\title{
Towards Generalizing Stochastic Spatiotemporal Graphs for Analyzing Least-Cost Path Stability
}

\author{
Chris J. Michael \\ chris.michael@nrlssc.navy.mil \\ U.S. Naval Research Laboratory \\ Stennis Space Center, Mississippi
}

\author{
Joseph P. Macker \\ joseph.macker@nrl.navy.mil \\ U.S. Naval Research Laboratory \\ Washington, DC
}

\author{
Frederick E. Petry \\ fred.petry@nrlssc.navy.mil \\ U.S. Naval Research Laboratory \\ Stennis Space Center, Mississippi
}

\begin{abstract}
In numerous applications, spatiotemporal graphs are studied to exploit the structure of underlying data to characterize, control, or predict behavior. Nodes of these graphs exhibit spatiality, while edge connectivity and weighting may be derived from spatial conditions of the incident nodes. Because of the complexity added by the temporal dimension, these graphs are typically modeled in part as a stochastic processes. Though numerous applicationdefined spatiotemporal graphs with stochastic parameterizations exist, a general stochastic process for such graphs has not yet been formally defined in the literature. In an effort to move towards generalization, we offer a brief introduction to the Stochastic SpatioTemporal (SST) graph model, which describes a graph as a set of initially observed nodes and several sets of stochastic processes: one describing node motion, one describing edge connectivity, and one describing edge weight variation. We propose a Monte Carlo method framework by which temporal graph algorithms that yield numerical or set-based results may be studied for conditions of stability. We demonstrate such a framework by way of a geometric SST graph, which is defined based on the geometric random graph exhibiting Brownian motion of nodes. We offer results to show the points at which node movement and edge weight variation cause the geometric SST graph to become unstable for predicting least-cost paths. Finally, we discuss ongoing research projects and plans currently being undertaken to study and utilize stochastic properties of spatiotemporal network data.
\end{abstract}

\section{KEYWORDS}

spatiotemporal data management, network theory, graph theory, spatiotemporal graph algorithms, stochastic processes

\section{ACM Reference Format:}

Chris J. Michael, Joseph P. Macker, and Frederick E. Petry. 2019. Towards Generalizing Stochastic Spatiotemporal Graphs for Analyzing Least-Cost Path Stability. In 31st International Conference on Scientific and Statistical Database Management (SSDBM '19), July 23-25, 2019, Santa Cruz, CA, USA. ACM, New York, NY, USA, 4 pages. https://doi.org/10.1145/3335783.3335807

\section{INTRODUCTION}

Stochastic analysis of spatiotemporal (ST) networks where nodes represent objects with spatiality are studied in numerous application areas such as mobile ad-hoc networks, motion planning, vehicle

This paper is authored by an employee(s) of the United States Government and is in the public domain. Non-exclusive copying or redistribution is allowed, provided that the article citation is given and the authors and agency are clearly identified as its source.

SSDBM '19, fuly 23-25, 2019, Santa Cruz, CA, USA

2019. ACM ISBN 978-1-4503-6216-0/19/07.

https://doi.org/10.1145/3335783.3335807 navigation, and signal propagation modeling. Graph-based analyses upon these types of networks are essential in order to characterize, control, and predict network behavior. For example, stochasticallydriven temporal graph analyses of mobile ad-hoc networks yield stable paths for message routing by analyzing the lifetime statistics of the network [9]. For underwater autonomous vehicle motion planning, ST graph algorithms are used for the online control of vehicle navigation given some set of mission goals and a probabilistic sampling of navigation points [10]. Traffic may be modeled stochastically in order to predict travel times and reliability in transportation networks [2]. Furthermore, protein interaction networks may be modeled as ST graphs to be studied statistically in order to predict signal propagation [7]. Though numerous applicationdriven models such as these exist, a general stochastic process for such graphs has not yet been formally defined in the literature.

Defining a general model for stochastic ST graphs is important for several reasons. First, a generalized stochastic process provides a natural framework by which large amounts of historic data may be used for analysis decoupled from a specific application. Second, the process defines a new random graph model for studying ST graphs where nodes are modeled as objects with spatiality and weighted directed edges represent spatially-dependent relationships between these objects. Finally, defining such a process provides a general framework for studying stability of temporal graph algorithms, as will be exemplified in this study.

A general model for graphs whose nodes exhibit spatiality were introduced in [5] where random plane networks, more commonly termed geometric random graphs, were defined. The nodes of these graphs exist on the Euclidean plane, while edges connect nodes within some circular radius in order to form components. Graphs whose edges encompass some type of stochastic weighting were introduced in [3], where a static graph's edges are labeled with cost and probability of traversal. The first model of temporal edge connectivity may have been [6], where temporally labeled edges are used to formulate the gossip problem. Since these, very many studies have been published on ST networks by combining one or some of these characteristics. A more recent effort to generally model ST graphs from object trajectories following from the geometric graph model was presented in [11]. Though this may be the closest study to our formulation, it differs from our goals in that it does not directly define a stochastic process.

We present a general stochastic process of ST graphs, named SST graph, that possesses all of the following properties: First, nodes have an initial spatial observation and exhibit movement with respect to a real-valued random variable. Second, directed edges connect nodes according to a random variable parameterized by a distance function upon the incident nodes. Finally, edge weights are 
set by a real-valued random variable parameterized by a distance function upon the incident nodes. To our knowledge, there is no such process defined in the current literature. We also present a framework for analyzing SST graph stability based on the MonteCarlo Method (MCM), upon which any temporal graph algorithms that yield numerical or set-based results may be studied. As an introductory demonstration, we define an SST graph based on the geometric random graph as well as a simple temporal LeastCost Path (LCP) algorithm. Finally, we use the MCM framework to analyze the stability of certain geometric SST graphs under two seperate conditions: One where node movement variation increases and edge weighting is based solely on incident node distances, and one where nodes remain static and edge weight variation increases. The results we present exemplify how one may use SST graphs and the MCM framework to study the thresholds at which parameters cause the system to become unstable.

Though the terms graph and network tend to be used interchangeably in much of the literature, we use network within the context of some application and graph within the context of general mathematical theory.

\section{SST GRAPH FORMULATION}

Define an SST graph $G=(N, \mathrm{X}, \mathrm{C}, \mathrm{W})$ where $N$ is a set of nodes that possess spatiality in $D$ dimensions, $\mathrm{X}$ is a set of random variables describing each node's movement, $\mathrm{C}$ is a set of random variables describing each possible edge's connectivity, and $\mathbf{W}$ is a set of random variables describing each edge's weighting. The coordinate position of each node $n \in N$ is determined by some observation $\hat{x}_{n o}=\left\{x_{n o 1}, \ldots, x_{n o D}\right\}$ that changes in time with respect to a set of real-valued random variables $\hat{\mathrm{X}}_{\mathrm{n}}=\left\{\mathrm{X}_{\mathrm{n} 1}, \ldots, \mathrm{X}_{\mathrm{nD}}\right\}$. These random variables may be viewed as a stochastic process representing node trajectories. A realization, or experiment, of the nodes over some given time interval $T$ yields the sets $\left\{\hat{\mathbf{X}}_{\mathbf{n}}(t)\right\}_{t \in T} \forall n \in N$. Let $E(t)$ be the set of edges in the realized graph at time $t$. An edge $e_{n, m}(t) \in$ $E(t)$ connecting node $n$ to node $m$ at time $t$ exists with respect to a boolean random variable $\mathrm{C}_{\mathbf{n}, \mathbf{m}}(t)$ where $e_{n, m}$ exists if and only if $\mathbf{C}_{\mathbf{n}, \mathbf{m}}(t)=1$. If such an edge exists, it is assigned a weight $w_{n, m}(t)$ with the real-valued random variable $\mathbf{W}_{\mathbf{n}, \mathbf{m}}(t)$. If it does not exist, then $w_{n, m}(t)=\infty$. It is important to highlight that $\mathbf{C}_{\mathbf{n}, \mathbf{m}}(t)$ and $\mathbf{W}_{\mathbf{n}, \mathbf{m}}(t)$ are dependent on $\hat{\mathbf{X}}_{\mathbf{n}}(t)$ and $\hat{\mathbf{X}}_{\mathbf{m}}(t)$.

In summary, an SST graph may be realized as an ST graph yielding the process $\{G(t)=(N(t), E(t))\}_{t \in T}$ in the following manner.

$$
\begin{gathered}
\hat{x}_{n}(1)=\hat{x}_{n o}+\hat{\mathbf{X}}_{\mathbf{n}}(1) \\
\hat{x}_{n}(t)=\hat{x}_{n}(t-1)+\hat{\mathbf{X}}_{\mathbf{n}}(\mathbf{t}) \\
w_{n, m}(t)=\left\{\begin{array}{l}
\mathbf{W}_{\mathbf{n}, \mathbf{m}}(t) \text { if } \mathbf{C}_{\mathbf{n}, \mathbf{m}}(t)=1 \\
\infty \text { otherwise }
\end{array}\right.
\end{gathered}
$$

Each node $n(t) \in N(t)$ is located at some coordinate position $\hat{x}_{n}(t) \in$ $\mathbb{R}^{D}$. Each edge $e_{n, m}(t)=(n(t), m(t)) \in E(t)$ has a weight $w_{n, m}(t) \in$ $\mathbb{R}_{\geq 0}$ or $\infty \forall t$. The chosen distributions of $\mathbf{C}_{\mathbf{n}, \mathbf{m}}$ and $\mathbf{W}_{\mathbf{n}, \mathbf{m}}$ are assumed to be parameterized with respect to some distance function $d\left(\hat{x}_{n}, \hat{x}_{m}\right)$, which we shorten as $d(n, m)$. For the purposes of this study, the spatiality of the ST graph is not used, so it will be referred to as a temporal graph. Therefore, this model is analogous to the time-aggregated graph described in [4].

\section{MCM FRAMEWORK}

A general framework for MCM-based stability analysis is now defined. Two types of analyses are covered: Those with numerical results and those where results are a set. Numerical analyses may be reduced using the following MCM framework given an SST graph.

$$
\begin{aligned}
f_{i} & =f\left(\{G(t)\}_{t \in T}\right) \forall i=[1, \ldots, k] \\
\bar{f} & =\frac{1}{k} \sum_{i=1}^{k} f_{i} \\
s_{f} & =\sqrt{\frac{1}{k-1} \sum_{i=1}^{k}\left(f_{i}-\bar{f}\right)^{2}}
\end{aligned}
$$

where $f$ is some function on a temporal graph yielding a numerical result, $T$ is the given time duration, $k$ is the given number of realizations, $\bar{f}$ is the calculated mean of the results of $f$ across all realizations, and $s_{f}$ is the calculated standard deviation of the results of $f$ across all realizations. For set-based results, the following MCM framework is proposed.

$$
\begin{aligned}
& F_{i}=F\left(\{G(t)\}_{t \in T}\right) \forall i=[1, \ldots, k] \\
& J_{f}=\left(\begin{array}{l}
k \\
2
\end{array}\right)^{-1} \sum_{i=1}^{k} \sum_{j=i+1}^{k} \frac{\left|F_{i} \cap F_{j}\right|}{\left|F_{i} \cup F_{j}\right|}
\end{aligned}
$$

where $F$ is some given function on a temporal graph yielding a set, and $J_{f}$ is the calculated mean Jaccard similarity taken across all realizations. Though several other possible solutions may be implemented within the framework, we focus on these two techniques for this introductory study. In summary, the results $s_{f}$ and $J_{f}$ may be used to analyze the stability of $f$ and $F$ respectively on the given SST graph $G$.

Before demonstrating the MCM framework, some formalizations are now made. Define a path as a sequence of edges

$$
\{p(h)\}_{h \in[1, H]}=\left\{e_{n_{0}, n_{1}}\left(t_{s}\right), e_{n_{1}, n_{2}}\left(t_{s}+1\right), \ldots, e_{n_{h-1}, n_{h}}\left(t_{e}\right)\right\}
$$

where any edge in the sequence $e(t) \neq \infty$ at time $t$ and the nodes along the path are not required to be unique. $H$ is the number of hops for the path. Define the aggregate weight of the path $p$ as $w_{p}$, which is calculated as the sum of the weights of corresponding edges in the sequence at their respective times. Because realizations of an SST graph are not guaranteed to yield one connected component, we choose to use the metric of path efficiency as $\epsilon_{p}=1 / w_{p}$. This metric was first introduced for weighted graphs in [8]. Efficiency, which in some contexts is called inverse geodesic distance, is a widely adopted property in applied graph theory due to its ability to characterize and compare local and global graph properties. Additionally, it offers a solution to the fundamental limitations that infinite-length paths introduce. Typically, the point-to-point path efficiencies are calculated for all pairs of nodes in a graph, and then the mean of all these values is taken to characterize the graph as a whole. This is referred to as the mean efficiency. Graphs with higher mean efficiencies exhibit higher connectivity. However, care must be taken when examining results comparatively due to the non-linearity of the reciprocal function.

LCP, or shortest path, algorithms are ubiquitous in graph analysis, and so we choose LCP as a first analysis for SST graphs. We 
define a temporal LCP algorithm, shown as Algorithm 1, to demonstrate the framework. This algorithm implements the LCP given a temporal graph $T G$, a source node $s r c$, a destination node $d s t$, and a time duration $T$ under the simplifying assumption that traversing any edge may be done in one time step. This algorithm is akin to the Bellman-Ford algorithm [1], but advances the temporal graph forward one time step for every edge relaxation step. The algorithm assumes that every node $n$ possesses a self edge with $w_{n, n}=0$. LCP weights to each destination node are stored in the map $\Delta$, while the nodes along the LCP to each destination node are stored in the map $p$. When used as a numerical analysis, the algorithm returns the LCP efficiency. When used as a set-based analysis, the algorithm returns the set of nodes along the LCP to the destination. Note that all values of $p$ are initialized to contain their own nodes as destinations. This avoids the undefined behavior that would occur from empty sets yielded when the LCP is not found.

The framework is exemplified through Algorithm 2 and Algorithm 3. Algorithm 2, which directly exemplifies the framework of the formulas shown in Formula (3), calculates LCP efficiency stability given the SST graph SSTG, the source node $s r c$, the destination node $d s t$, the time duration $T$, and the number of realizations $k$. It does so by returning the mean and standard deviation of the LCP efficiency across all $k$ realizations of the SST graph. Likewise, Algorithm 3 directly exemplifies the framework of Formula (4) to calculate the mean of all LCP similarities across the $k$ realizations.
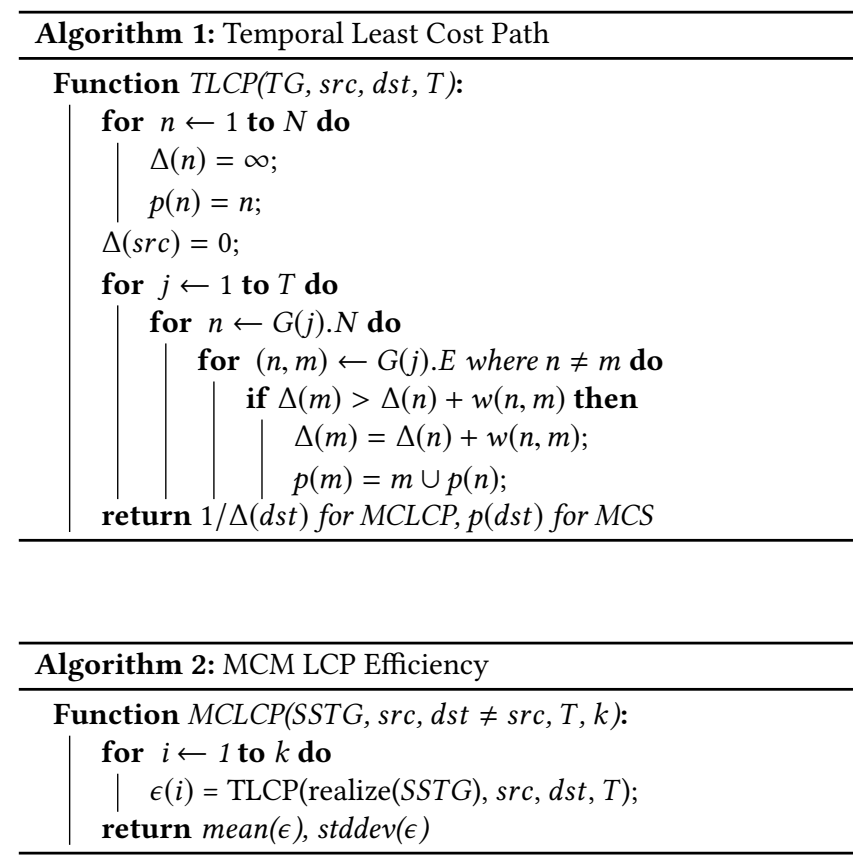

\section{DEMONSTRATION}

A synthetic SST graph is created based on nodes exhibiting Brownian motion whose connectivity model follows that of the geometric random graph with the number of dimensions $D=2$. In order to simplify the demonstration, we fix certain stochastic variables as constant. Define the Geometric SST graph $G=(N, \mathbf{X}, \mathrm{C}, \mathbf{W})$ where

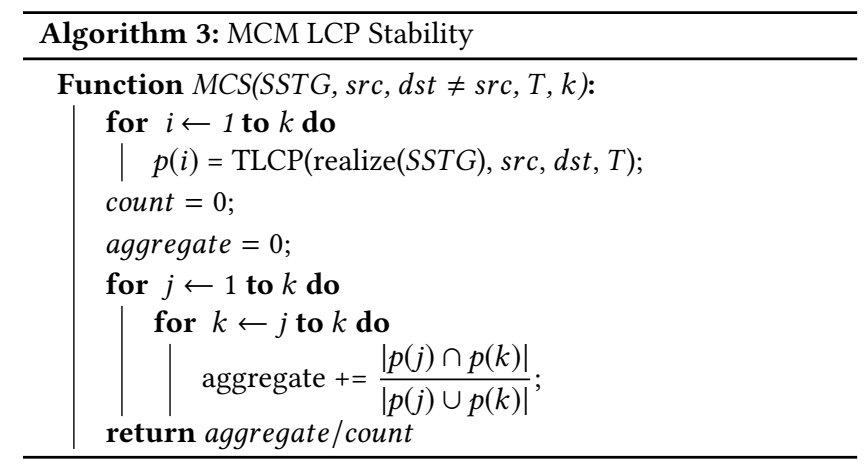

$N$ is a set of nodes, each with initial position $x_{n o 1}=\mathcal{U}(0,100)$ and $x_{n o 2}=\mathcal{U}(0,100)$ with $\mathcal{U}(0,100)$ representing a uniformly distributed real-valued random variable with range $[0,100]$. For the set $\mathrm{X}$, set each $\hat{\mathrm{X}}_{\mathbf{n}}=\left\{\mathcal{N}\left(0, \sigma_{n}\right), \mathcal{N}\left(0, \sigma_{n}\right)\right\}$ where $\mathcal{N}$ is a Gaussian random variable and $\sigma_{n}=\mathcal{U}\left(0, \sigma_{n}^{\max }\right)$. Take the set $\mathrm{C}$ to consist of all $\mathbf{C}_{\mathbf{n}, \mathbf{m}}(t)$ where the probability $P\left(\mathbf{C}_{\mathbf{n}, \mathbf{m}}(t)=1\right)=1$ if $d(n(t), m(t))<=r$ and $P\left(\mathbf{C}_{\mathbf{n}, \mathbf{m}}(\mathbf{t})=1\right)=0$ otherwise. This property mimics that of the geometric random graph in that a node is connected to all other nodes within a circle of radius $r$ (except that edges are not removed to form the connected component as in the original study). The set $\mathbf{W}$ consists of all $\mathbf{W}_{n, m}(t)=\mathcal{N}\left(d(n(t), m(t)), \sigma_{n, m}\right)$ where values for $\sigma_{n, m}=\mathcal{U}\left(0, \sigma_{n, m}^{\max }\right)$. Because it is possible that edge weights may be negative for high values of $\sigma_{n, m}^{\max }$, we take the absolute values of all edge weights derived by the process. This is a minor adjustment since only two series of experiments produced negative edge weights, and the changes in stability induced by taking the absolute value were trivial.

Though it may be intuitive that stability of the SST graph decreases as variations in node movement and edge weight increases, the point at which these variations cause the graph to be unstable is not intuitive. Therefore, we provide empirical results with the aim of finding the point at which this phase change occurs for a chosen geometric SST graph. For all experiments, we set $k=100$, $|N|=50, r=20$, and $T=30$. We chose these parameters to be manageable yet to yield interesting graph configurations such as those studied in wireless communication networks.

Algorithms 2 and 3 are modified to be single-to-all instead of point-to-point to reflect the entire graph's stability with respect to a given source node. Algorithm 2 will calculate the LCP mean efficiency from the source node to all other nodes for each realization of the SST graph. Then, the mean and standard deviation of this result is calculated across all realizations. For Algorithm 3, the set of nodes comprising the LCP from the source node to every other node in the graph are calculated. Then, the similarity of this result is calculated across all realizations. Finally, the average of these similarity indexes is taken to provide the results. Experiments we conducted separately have shown that the difference in results for single-to-all and all-to-all LCP are trivial for the geometric SST graph. We choose single-to-all for simplicity in formulating the result while reflecting the entire graph's stability.

The stability results for various values of $\sigma_{n}^{\max }$ while setting $\sigma_{n, m}^{\max }=0$ are shown in Figure 1. In this experiment, edge weights change with respect to node movement alone. Plots a and b show 


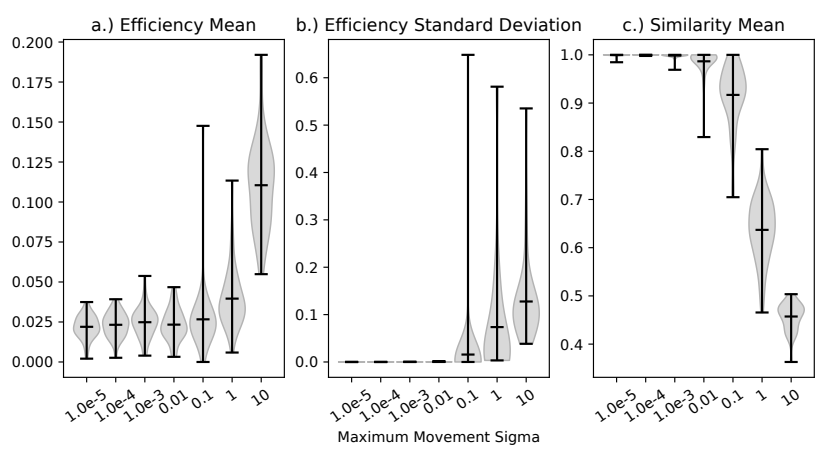

Figure 1: Movement Stability

results for efficiency, while Plot c shows results for path similarity. Each value on the $\mathrm{x}$-axis denotes an experimental series for which $\sigma_{n}^{\max }$ is set. Within each series, 50 experiments are conducted for the described geometric SST graph with all parameters other than $\sigma_{n}^{\max }$ fixed as mentioned before. The shaded region within each series shows the distribution of results within these experiments. The vertical bar with tick marks denote the maximum, mean, and minimum points for these results. Be reminded that efficiency standard deviations naturally diminish for lower efficiencies due to the reciprocal function on path weights. The results for LCP similarity do not diminish in this manner.

In Figure 1a, the mean path efficiency tends to increase as the node movement variation increases. This phenomenon occurs because larger magnitude movement of nodes tends to increase the likelihood of a more efficient path to be found. However, as can be shown in Figure $1 \mathrm{~b}$ and especially Figure 1c, the LCPs tend to show more efficiency variation and much less path similarity. One may easily draw the conclusion that the geometric SST graph as parameterized exhibits predictable behavior for calculating LCP up to $\sigma_{n}^{\max }=0.1$, after which the graph becomes unstable and hence unpredictable. The results for stability under edge weight variation are shown in Figure 2. In these experiments, node positions are fixed while $\sigma_{n, m}^{\max }$ is increased. Because the nodes do not move, the graph realizations generally exhibit less LCP efficiency and higher stability in general when compared to Figure 1. Here, as can be shown in Plots b and c, mean path efficiencies and path similarities may be ruled as stable until $\sigma_{n, m}^{\max }=10$.

\section{DISCUSSION AND ONGOING RESEARCH}

We have formally defined the SST graph and given an example based on Brownian motion and the random geometric graph model. The framework introduced in this study allows for stability of general-purpose temporal graph algorithms to be studied through the stochastic process inherent to SST graphs.

The purpose of this study is to introduce SST graphs and offer a preliminary demonstration of how they may be used to characterize complex relational spatiotemporal data. The MCM framework, though shown to be effective, requires tremendous amounts of computation for more complex analyses. We are currently studying optimization and search algorithms that more quickly find the
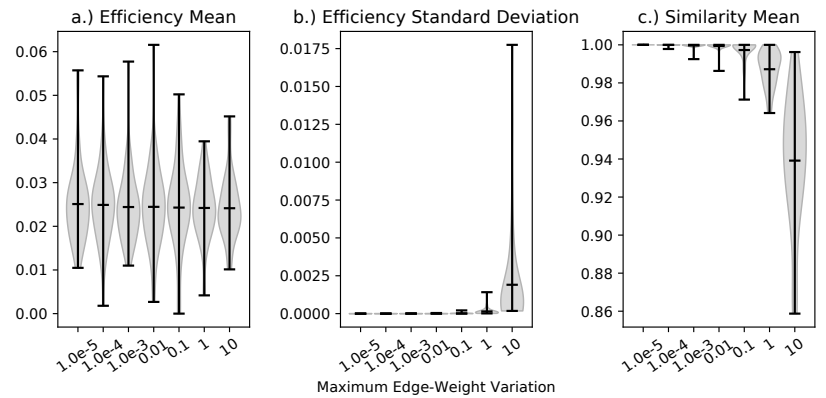

Figure 2: Edge-Weight Variation Stability

parameterizations at which SST graphs lose stability for several chosen algorithms.

There are several broader research studies we are undertaking to further generalize stochastic properties of spatiotemporal data. Data across several real-world network applications are being collected into a benchmark suite to study the effectiveness of using SST graphs to predict behavior through statistical inference. Parallel processing paradigms for better scaling are being explored and implemented within the software architecture used to define and instantiate SST graphs. For instance, we are incorporating novel spatiotemporal search algorithms to speed up and parallelize the spatial query involved with setting the connectivity from $\mathrm{C}$. There are also efforts being undertaken to study simplification of spatial behavior through likelihood functions so that the harrowing volume of data generated by ST networks may be reduced to focus on those specific behaviors that are of importance.

\section{REFERENCES}

[1] Richard Bellman. 1958. On a routing problem. Quarterly of applied mathematics 16, 1 (1958), 87-90.

[2] Bi Yu Chen, William HK Lam, Agachai Sumalee, and Zhi-lin Li. 2012. Reliable shortest path finding in stochastic networks with spatial correlated link travel times. International fournal of Geographical Information Science 26, 2 (2012), 365-386.

[3] John S Croucher. 1978. A note on the stochastic shortest-route problem. Naval Research Logistics Quarterly 25, 4 (1978), 729-732.

[4] Betsy George and Shashi Shekhar. 2008. Time-aggregated graphs for modeling spatio-temporal networks. In fournal on Data Semantics XI. Springer, 191-212.

[5] Edward N Gilbert. 1961. Random plane networks. F. Soc. Indust. Appl. Math. 9, 4 (1961), 533-543.

[6] Frits Göbel, J Orestes Cerdeira, and Hendrik Jan Veldman. 1991. Label-connected graphs and the gossip problem. Discrete Mathematics 87, 1 (1991), 29-40.

[7] Chittaranjan Hens, Uzi Harush, Simi Haber, Reuven Cohen, and Baruch Barzel. 2019. Spatiotemporal signal propagation in complex networks. Nature Physics (2019), 1.

[8] Vito Latora and Massimo Marchiori. 2001. Efficient behavior of small-world networks. Physical review letters 87, 19 (2001), 198701.

[9] Joseph P Macker, David Claypool, and Nathan Hughes. 2014. Improving Routing Path Stability in Mobile Ad Hoc Networks That Use a CDS Control Plane. In 2014 IEEE Military Communications Conference. IEEE, 1099-1104.

[10] James McMahon and Erion Plaku. 2016. Mission and motion planning for autonomous underwater vehicles operating in spatially and temporally complex environments. IEEE Journal of Oceanic Engineering 41, 4 (2016), 893-912.

[11] Tilemachos Pechlivanoglou and Manos Papagelis. 2018. Fast and Accurate Mining of Node Importance in Trajectory Networks. In 2018 IEEE International Conference on Big Data (Big Data). IEEE, 781-790. 